\title{
Photoproduction models for total cross section and shower development
}

\author{
Fernando Cornet $^{1, \mathrm{a}}$, Carlos Garcia Canal $^{2, \mathrm{~b}}$, Agnes Grau ${ }^{1, \mathrm{c}}$, Giulia Pancheri ${ }^{3, \mathrm{~d}}$, and Sergio Sciutto ${ }^{2, \mathrm{e}}$ \\ 1 Departamento de Física Teórica y del Cosmos and Centro Anadaluz de Física de Partículas, Universidad de Granada, \\ 18071 Granada, Spain \\ 2 Departamento de Física, Universidad Nacional de La Plata, IFLP, CONICET, C.C.67, 1900 La Plata, Argentina \\ 3 INFN Frascati National Laboratories, via E. Fermi 40, 00044 Frascati, Italy
}

\begin{abstract}
A model for the total photoproduction cross section, based on the ansatz that resummation of infrared gluons limits the rise induced by QCD minijets in all the total cross-sections, is used to simulate extended air showers initiated by cosmic rays with the AIRES simulation program. The impact on common shower observables, especially those related with muon production, is analysed and compared with the corresponding results obtained with previous photoproduction models.
\end{abstract}

\section{Introduction}

Cosmic rays have often anticipated discoveries in particle physics and have allowed exploration of energies which were not available by particle accelerators at the time. Such was the case of the rise of the total $p p$ cross-section in the early '70s [1], an observation confirmed shortly after by ISR [2]. The rise of all hadronic cross-sections was soon suggested to be due to an increasing number of parton-parton collisions [3], which can be described by perturbative QCD and are referred to as mini-jets. An early application of an eikonalized mini-jet model for cosmic ray interactions [4] still reproduces within errors the recent AUGER measurement of the total $p-$ air cross-section [5]. Different models for the basic $p p$ interaction and its applications to cosmic rays are discussed in the literature, and included in MonteCarlo simulations, many of them using mini-jets as the driving phenomenon of the rise. Mini-jet models have been extended to photoproduction, with some additional parameterisation of the parton content of the photon, e.g. in [6,7] and [8].

In this contribution we shall study shower observables in cosmic ray photo production obtained by two different models for the total $\gamma-p$ cross-section as input to the AIRES MonteCarlo [9], the eikonalized mini jet model with soft gluon resummation of [8] and the analytic amplitude model by Block and Halzen [10], which is presently the one used in AIRES. We shall compare predictions at very high energies, where QCD processes are expected to be dominant, and highlight the differences in different observables.

\footnotetext{
a e-mail: cornet@ugr.es

b e-mail: cgarciacanal@fisica.unlp.edu.ar

${ }^{c}$ e-mail: igrau@ugr.es

d e-mail: pancheri@lnf.infn.it

e e-mail: sciutto@fisica.unlp.edu.ar
}

\section{Photoproduction mini-jet model for total cross section}

In order to study $\gamma-$ air at very high energies, we first update the results of the eikonal mini-jet model for $\gamma-p$ interaction of [8] in the light of the recent LHC results for the total $p p$ cross-section [11-13].

We shall start with a brief summary of the content of the model for $p p$ and its extension to $\gamma-p$.

Mini-jet models for the total cross-section use as input a perturbative calculation based on the QCD jet crosssection, namely

$$
\begin{aligned}
\sigma_{\text {jet }}^{A B}\left(s, p_{\text {tmin }}\right) & =\int_{p_{t \text { min }}}^{\sqrt{s} / 2} d p_{t} \int_{4 p_{t}^{2} / s}^{1} d x_{1} \int_{4 p_{t}^{2} /\left(x_{1} s\right)}^{1} d x_{2} \times \\
& \times \sum_{i, j, k, l} f_{i \mid A}\left(x_{1}, p_{t}^{2}\right) f_{j \mid B}\left(x_{2}, p_{t}^{2}\right) \frac{d \hat{\sigma}_{i j}^{k l}(\hat{s})}{d p_{t}}
\end{aligned}
$$

with $A, B=p, \bar{p}, \gamma$. The parton-parton differential crosssection $d \hat{\sigma}_{i j}^{k l}(\hat{s}) / d p_{t}$ defines the total contribution for partons with $p_{t}>p_{\text {min }}$, where the parameter $p_{\text {tmin }} \approx$ $1-2 \mathrm{GeV}$ separates hard processes for which one can use a perturbative QCD description, from the soft ones which dominate at low c.m. energy of the scattering hadrons, $\sqrt{s} \lesssim 10 \div 20 \mathrm{GeV}$. The mini-jet cross-section gets its name because it is dominated by low- $p_{t}$ processes, which cannot be identified by jet finding algorithms, but can still be perturbatively calculated using partonparton sub-processes and DGLAP evoluted LO Partonic Density Functions $f_{i \mid A}$. The expression of Eq. (1) gives a mini-jet cross-section which rises very fast with energy. In order to ensure unitarity, it is embedded in an eikonal representation, which requires modeling the impact parameter space of the colliding hadrons. Neglecting the real part of the eikonal, which at high

This is an Open Access article distributed under the terms of the Creative Commons Attribution License 4.0, which permits unrestricted use, distribution, and reproduction in any medium, provided the original work is properly cited. 
energy is a good approximation, one then writes

$$
\sigma_{\text {tot }}=2 \int d^{2} b\left[1-e^{-\chi_{I}(b, s)}\right] .
$$

In these models, the imaginary part of the eikonal function, $\chi_{I}$, is calculated from the average number of collisions as $n(b, s)=2 \chi_{I}(b, s)=n_{\text {soft }}(b, s)+n_{\text {hard }}(b, s)$, namely it is split into a soft contribution which will be parametrized with a suitable non-perturbative expression, and a perturbative (pQCD) term where both hard and soft gluon emission contribute. The mini-jet model of [8], called Bloch Nordsieck (BN) model, proposes two different impact parameter distributions for these two terms, i.e.

$$
\begin{gathered}
n_{\text {soft }}(b, s)=A_{F F}(b, s) \sigma_{\text {soft }}(s) \\
n_{\text {hard }}(b, s)=A_{B N}(b, s) \sigma_{\text {jet }}(s)
\end{gathered}
$$

where $A_{F F}$ is obtained from the proton form factor, and $A_{B N}(b, s)$ as the Fourier transform in impact parameter space of the function $d^{2} P\left(\mathbf{K}_{\perp}\right) / d^{2} \mathbf{K}_{\perp}$ obtained as resummation of all soft gluons, emitted in parton-parton collisions with $p_{t}>p_{\text {tmin }}$, down into the infrared region [14], i.e.

$$
\begin{array}{r}
A_{B N}(b, s)=N \int d^{2} \mathbf{K}_{\perp} e^{-i \mathbf{K}_{\perp} \cdot \mathbf{b}} \frac{d^{2} P\left(\mathbf{K}_{\perp}\right)}{d^{2} \mathbf{K}_{\perp}}= \\
=\frac{e^{-h\left(b, q_{\max }\right)}}{\int d^{2} \mathbf{b} e^{-h\left(b, q_{\max }\right)}} .
\end{array}
$$

The function $h\left(b, q_{\max }\right)$ corresponds to the spectrum for single gluon emission, regularized in the infrared. The extension to infrared gluons is crucial for this model and requires an ansatz as to the coupling of very soft gluons to the emitting quarks, which the model introduces through a singularity parameter $1 / 2<p<1$ [14]. The physical content of this model can be summarized as follows: i) the rise is obtained from low-x parton-parton collision, ii) the taming of the rise is obtained from the Fourier transform in impact parameter space of the resummation of all soft gluons emitted in the parton-parton collisions. The expression for the impact parameter distribution when the two hadrons collide describes the acollinearity introduced by soft gluon emission which reduces the cross-section from mini-jets. The expression is energy dependent through the parameter $q_{\max }$, which embeds the kinematics of single gluon emission, and represents the maximum transverse momentum for single gluon emission and depends on the $p_{t}$ of the final state partons description of how to calculate $q_{\max }$ for purely proton processes can be found in as detailed in [14] for $p p$ collisions. Through the ansatz for the coupling in the infrared region, the distribution $A_{B N}(b, s)$ gives a (logarithmically) energy dependent cut-off in b-space, dynamically generated by soft gluon emission, and reduces the very fast rise from the mini-jet cross section [15].

With the above inputs, and a parametrization of the low energy region, i.e. $n_{\text {soft }}(b, s)$, the model gave a good description of $p p$ and $p \bar{p}$ total cross-sections, predicting $\sigma(\sqrt{s}=14 \mathrm{TeV})=100 \pm 12 \mathrm{mb}$, with the error to reflect various uncertainties in choice of parton densities and

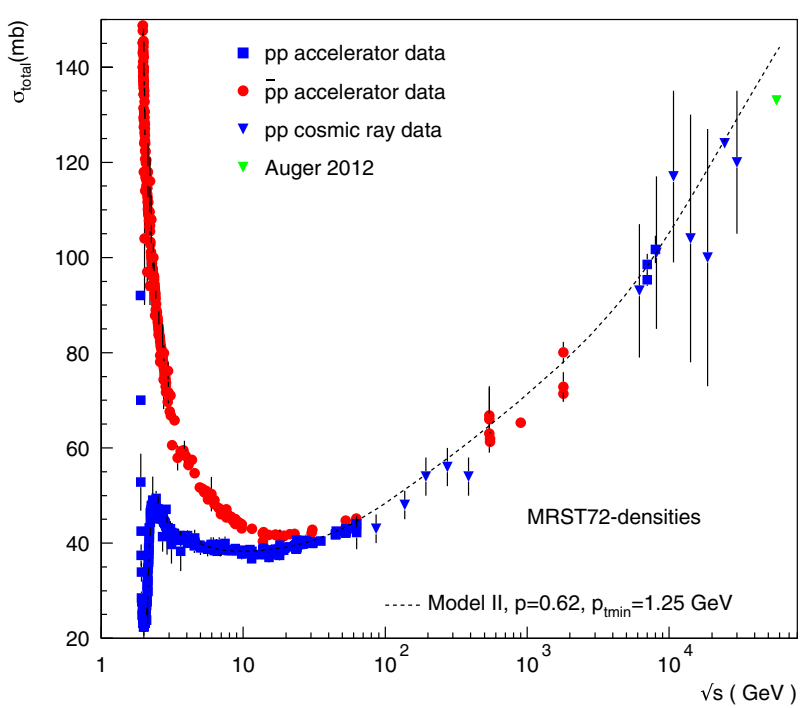

Figure 1. Total proton-proton cross-section and its description with the BN model, eikonalized mini-jets with soft gluon resummation, described in the text.

values for the set of non-perturbative parameters $\left\{p, p_{t \min }\right\}$ [14]. Following [6], this model was then applied to photoproduction [8], namely

$$
\begin{array}{r}
\sigma_{\text {tot }}^{\gamma p}=2 P_{\text {had }} \int d^{2} b\left[1-e^{-n^{\gamma p}(b, s) / 2}\right] \\
P_{\text {had }}=\sum_{V=\rho, \omega, \phi} \frac{4 \pi \alpha}{f_{V}^{2}} \\
n^{\gamma p}(b, s)=n_{\text {soft }}^{\gamma p}(b, s)+n_{\text {hard }}^{\gamma p}(b, s) \\
=n_{\text {soft }}^{\gamma p}(b, s)+A(b, s) \sigma_{\text {jet }}^{\gamma p}(s) / P_{\text {had }} \\
n_{\text {sof } t}^{\gamma p}(b, s)=\frac{2}{3} n_{\text {soft }}^{\text {pp }}(b, s) .
\end{array}
$$

The extension to photon process requires the probability $P_{\text {had }}$ that the photon behaves like a hadron $[6,16]$. This quantity is non perturbative and could have some mild energy dependence. However, to minimize the parameters, it was taken to be a constant, estimating it through Vector Meson Dominance.

With such modifications, one can now calculate the $\gamma p$ cross-section that will be used as an input in the AIRES shower simulation program. To determine the parameter sets which best describe the behavior at energies as high as those reached by cosmic rays, one should take into account the impact of recent LHC [11-13] and AUGER Observatory [5] results on the $p p$ cross-section, which have appeared after the original analysis of [8]. Indeed, the measurement of the total $p p$ cross-section at LHC, at energies $\sqrt{s}=7$ and $8 \mathrm{TeV}$, has allowed to reduce the systematic uncertainties present in most models, due to the large errors affecting the $\bar{p} p$ cross-section. We show in Fig. 1 how this model accommodates recent results for $\sigma_{\text {total }}^{p p}$, including the extraction of the $p p$ crosssections from cosmic ray measurement by the AUGER collaboration, at $\sqrt{s}=57 \mathrm{TeV}$. With the choice of parameters as indicated in the figure and MRST72 densities [17], the value expected at $\sqrt{s}=14 \mathrm{TeV}$ is 


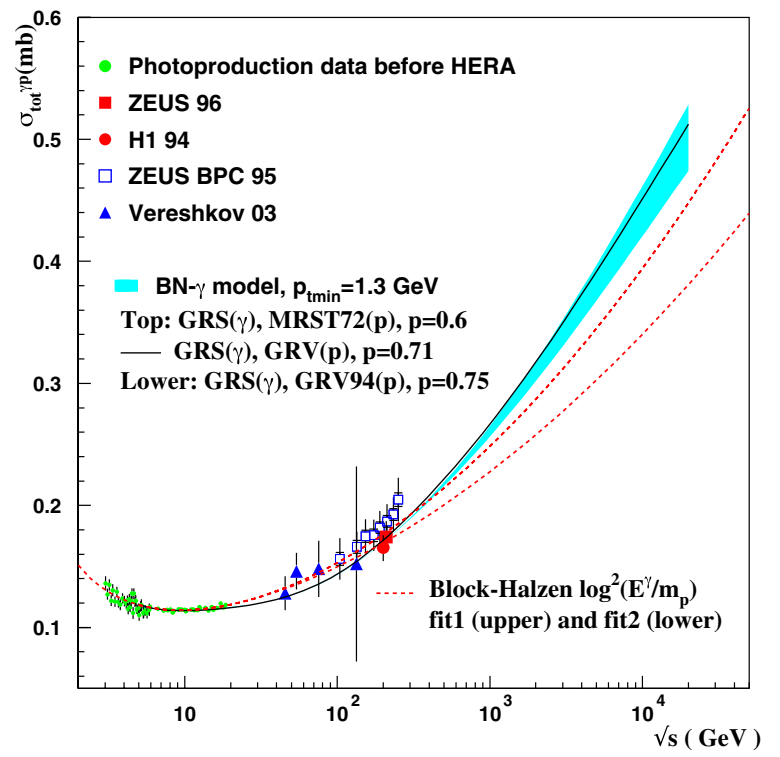

Figure 2. Total photoproduction cross-section and its description with the BN model, and with the analytical models by Block and collaborators [10].

$\sigma_{\text {total }}^{p p}=112.24 \mathrm{mb}$. Using other types of LO densities, such as the older GRV [18] or more recent MSTW [19], gives similar values [20], namely, up to LHC values, the predictions are rather stable, for different densities, once the TOTEM and AUGER points are included in the description.

The extension of the model to $\gamma-p$ is shown in Fig. 2, where GRS are the LO PDFs for the photon [21]. In the figure the results from the $\mathrm{BN}$ model are compared with results from two fits by Block and collaborators, which impose a Froissart-limit saturating high energy behavior [10].

Before moving to $\gamma$-air nucleus simulations, we notice that, at higher c.m. values where accelerator data are not available, the model we have described gives predictions which differ by $10-20 \%$, depending on the PDFs used, and also different from both fits of Ref. [10]. For our model, the difference corresponds to the different PDFs used, and thus to the different low-x behavior of these densities.

\section{Observables in shower development}

We now apply the above model to study atmospheric air showers initiated by photons. For these simulations, we use the cross-section that corresponds to the solid line within the blue band in Fig. 2 and fit 2 of [10]. We shall see how changes in the $p p$ cross-section at high energy affect the photo-nuclear cross-section at the corresponding energies, and, through this, photon initiated shower observable. In such showers, the photonuclear reactions constitute the main channel for production of hadrons. These hadrons are responsible for the production of muons, mainly via pion decay. It is a well known fact that showers initiated by photons have noticeably less muons than showers initiated by hadrons, and this is one

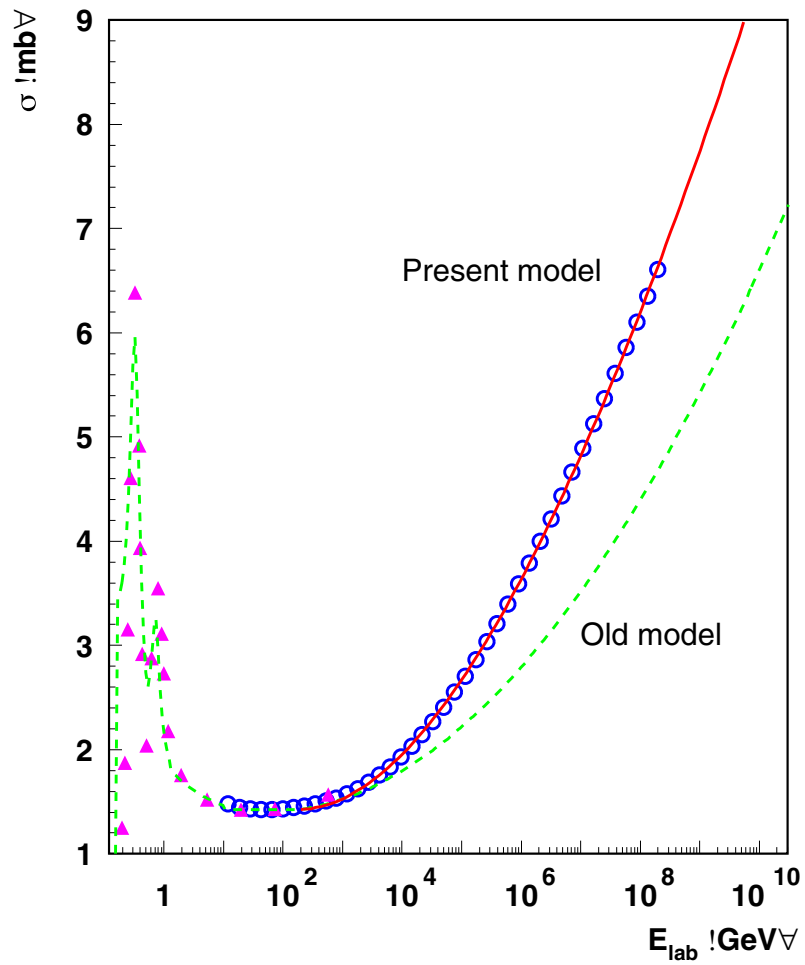

Figure 3. Photon-air nucleus cross sections, used in the present simulations, plotted versus photon lab energy. The triangles correspond to experimental data taken from reference [23]. The open circles correspond to the present model (Eq. (7)), and the solid line corresponds to a fit to these points, valid for energies greater than $200 \mathrm{GeV}$. The dashed line corresponds to the cross sections used in AIRES.

of the features used to discriminate photon initiated from hadron initiated showers.

We have performed simulations of extended air showers using the AIRES system [9] together with the hadronic interaction package QGSJET-II [22]. We have run two sets of simulations, namely, (1) using the cross sections for photonuclear reactions at energies greater than $200 \mathrm{GeV}$ that are provided with the currently public version of AIRES; and (2) replacing those cross sections by the ones corresponding to the present model. We are going to refer to sets (1) and (2) as old model and present model, respectively.

In Fig. 3 the different $\gamma$-air nucleus cross sections are displayed as a function of photon lab energy.

The triangles correspond to experimental data taken from [23], while the open circles correspond to numerical calculations starting from Eq. (7). The solid line is a fit to the results from the above model, valid for energies greater than $200 \mathrm{GeV}$. The dashed line corresponds to the up to now standard cross sections implemented in AIRES, the old model. Notice that for energies less than $200 \mathrm{GeV}$ we use always the same cross sections, which are calculated from fits to experimental data.

Figure 3 shows that at energies $E_{L A B} \sim 10^{19} \mathrm{eV}$ and beyond, there is a large difference, more than $50 \%$, between the results for the two models and one can expect this to reflect in some of the observables in the photon 


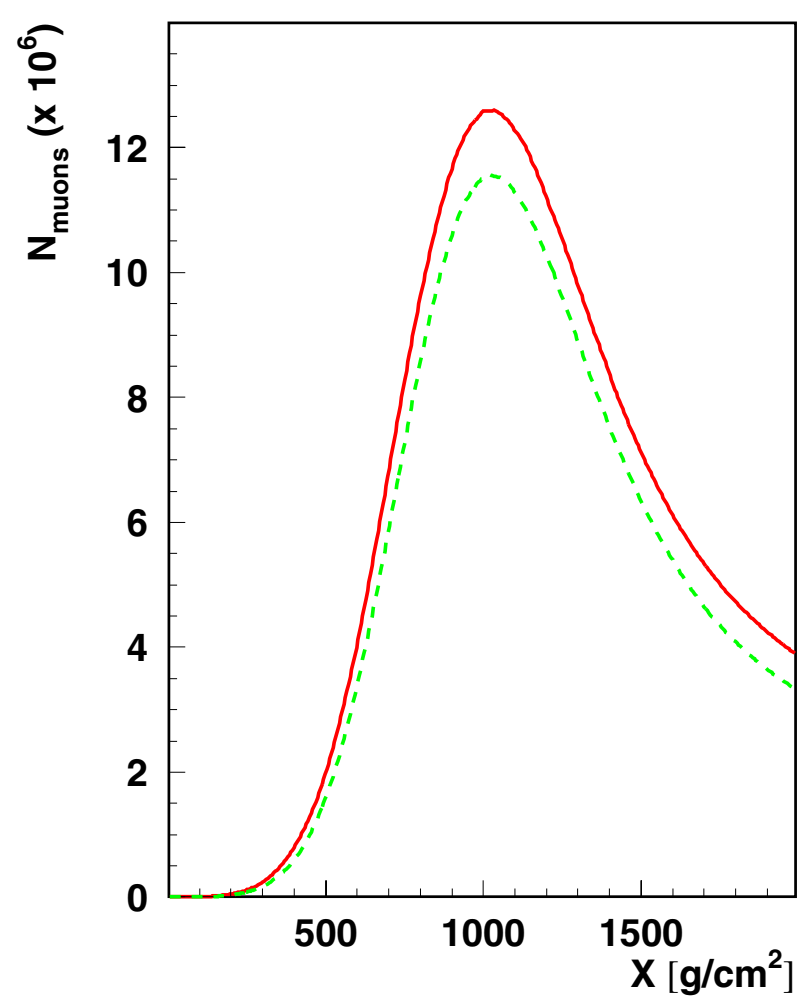

Figure 4. Longitudinal development of muons for $10^{19} \mathrm{eV}$ photon showers inclined 60 degrees. The solid (dashed) line corresponds to simulations with the present (old) model for photonuclear crosssections.

initiated showers. For reasons of brevity, in this contribution we present results only for this very representative case, of $10^{19} \mathrm{eV}$ gamma showers. At this primary energy, geomagnetic conversion is not frequent, thus allowing photons to enter the atmosphere unconverted, and initiate normally the shower development. We have taken in our simulations a ground altitude of 1400 meters above sea level (m.a.s.l.), corresponding to the altitude of current Cosmic Ray Observatories. The most probable photon interactions at the mentioned energy are electromagnetic (i.e., pair production), and for that reason most of the shower secondaries will always be electrons and photons; and the number of such secondaries is not expected to change substantially when replacing the photonuclear cross sections. On the other hand, we can expect differences in muon showers, since muons are produced by the decay of unstable hadrons, whose production is affected by the hadronic model for the interaction. It is also important to consider the characteristics of the muons produced in the simulations. Let us focus on the representative case of $10^{19} \mathrm{eV}$ gamma vertical showers with ground altitude 1400 m.a.s.l. This corresponds roughly to an atmospheric slant depth of $900 \mathrm{~g} / \mathrm{cm}^{2}$. We present our results for the longitudinal development of muons in Fig. 4, where it shows up clearly that the simulations with present model produce more muons in virtually the entire shower life. The relative difference with respect to the old model is about $12 \%$ at the maximum $\left(X \simeq 1100 \mathrm{~g} / \mathrm{cm}^{2}\right)$. Accordingly with the results displayed in Fig. 4 this depth is located short before the maximum of the muon

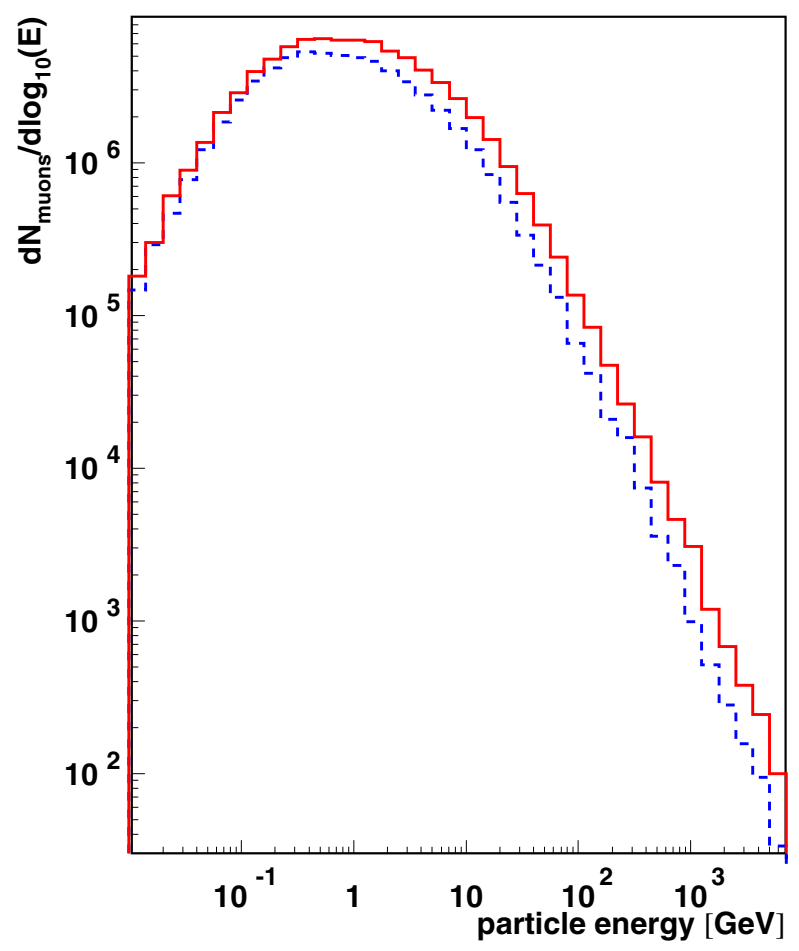

Figure 5. Energy distribution of ground muons. The solid (dashed) line corresponds to simulations with the present (old) model for photonuclear cross sections.

longitudinal profile. Concerning the lateral distribution of muons, not much difference is noticed between the two models, the shape is very similar, and the results differ only in the total number of particles. On the other hand, the muon energy distributions displayed in Fig. 5 present noticeable differences for muon energies greater than roughly $1 \mathrm{GeV}$, with the present model giving the largest number of particles at each bin. For muon energies less than $1 \mathrm{GeV}$, both distributions are virtually coincident.

Since shower muons are generated after the decay of unstable hadrons, mainly charged pions and kaons, the enlarged number of muons that show up in Fig. 4 would be necessarily connected with enlarged hadron production. The results of our simulations agree with this expected behaviour.

\section{Final remarks}

The main objective of this contribution is to present a QCD-based model for photoproduction, updated from the previous analysis [8] in light of recent LHC results for total $p p$ cross-sections, and to study the impact of this new model on the air shower development. At very high energies, this model produces a photon-air nucleus total cross section significantly larger than the previous models included in the standard extended air shower studies.

The present analysis based on simulations by means of the AIRES system clearly shows that for photon initiated showers the total muon production is increased in a measurable way. This result could be of direct importance in future determinations of bounds for the highest energy cosmic photon flux. In this respect, a more detailed analysis of this kind of effects is in progress. 
This collaboration was partially financed by the Programa de Cooperación Científico Tecnológico Argentino-Español: MinCyT-MINCINN. F.C. and A.G. acknowledge financial support from Junta de Andalucía (FQM-330, FQM-101, FQM6552) and MICINN projects FPA2010-16696 and ConsoliderIngenio 2010 program CPAN (CSD2007-00042). Partial support by CONICET and ANPCyT, Argentina is acknowledged.

\section{References}

[1] G.B. Yodh, Y. Pal and J.S. Trefil, Phys. Rev. Lett. 28, 1005 (1972)

[2] U. Amaldi et al. Phys. Lett. B44, 112 (1973)

[3] D. Cline, F. Halzen and J. Luthe, Phys. Rev. Lett. 31, 491 (1973)

[4] L. Durand, H. Pi, Phys.Rev. D38, 78-84 (1988)

[5] P. Abreu, et al., Phys. Rev.Lett. 109, 062002 (2012)

[6] R.S. Fletcher, T.K. Gaisser and F. Halzen, Phys. Lett. B298, 442 (1993); Phys. Rev. D45, 377-381 (1992), Erratum-ibid.D45 3279 (1992)

[7] M.M. Block, E.M. Gregores, F. Halzen, G. Pancheri, Phys.Rev. D60, 054024 (1999)

[8] R.M. Godbole, A. Grau, G. Pancheri and Y.N. Srivastava, Eur. Phys. J.C63, 69-85 (2009)

[9] AIRES User's Manual and Reference Guide, available electronically at www2.fisica.unlp.edu. ar/auger/aires

[10] M.M. Block, L. Durand, P. Ha, Phys. Rev. D89, 094027 (2014)

[11] Antchev, et al., Europhys.Lett. 101, 21004 (2013)
[12] G. Antchev, et al., Phys.Rev.Lett. 111, 012001 (2013)

[13] ATLAS Collaboration, Nucl. Phys. B889, 486-548 (2014)

[14] A. Corsetti, A. Grau, G. Pancheri and Y.N. Srivastava, Phys. Lett B382, 282 (1996); A. Grau, G.Pancheri, Y.N. Srivastava, Phys. Rev. D60, 114020 (1999); R. M. Godbole, A. Grau, G. Pancheri and Y. N. Srivastava, Phys. Rev. D72, 076001 (2005)

[15] A. Grau, R.M. Godbole, G. Pancheri and Y.N. Srivastava, Phys. Lett. B682, 55-60 (2009)

[16] J. C. Collins, G.A. Ladinsky, Phys.Rev. D43 28472855 (1991)

[17] A.D. Martin, R.G. Roberts, W. J. Stirling, and R.S. Thorne, Eur. Phys. J. C4, 463 (1998); A. D. Martin, R. G. Roberts, W. J. Stirling, and R. S. Thorne, Phys. Lett. B531, 216-224 (2002)

[18] M. Gluck, E. Reya, and A. Vogt, Z. Phys. C53, 127-134 (1992); Z. Phys. C67, 433-448 (1995); Eur. Phys. J. C5 461-470 (1998)

[19] A.D. Martin, W.J. Stirling, R.S. Thorne and G. Watt, Eur. Phys. J. C63 189 (2009)

[20] D.A. Fagundes, A. Grau, G. Pancheri, Y.N. Srivastava and O. Shekhovtsova, e-print: arXiv:1408.2921 [hep-ph]

[21] M. Gluck, E. Reya and I. Schienbein, Phys. Rev. D60, 054019 (1999); Erratum, ibid D62, 019902 (2000)

[22] S. Ostapchenko, Phys. Rev. D83, 014018 (2011)

[23] K.A. Olive et al. (Particle Data Group), Chin. Phys. C38, 090001 (2014) 